\title{
Seidel Laplacian and Seidel Signless Laplacian Spectrum of the Zero-divisor Graph on the Ring of Integers Modulo $n$
}

\author{
Magi P $\mathbf{M}^{1, *}$, Sr.Magie Jose ${ }^{1}$, Anjaly Kishore ${ }^{2}$ \\ ${ }^{1}$ Department of Mathematics, St.Mary's College, Thrissur, Kerala, India \\ ${ }^{2}$ Department of Mathematics, Vimala College, Thrissur, Kerala, India
}

Received June 1, 2021; Revised October 11, 2021; Accepted October 21, 2021

Cite This Paper in the following Citation Styles

(a): [1] Magi P M, Sr.Magie Jose, Anjaly Kishore, ”Seidel Laplacian and Seidel Signless Laplacian Spectrum of the Zero-divisor Graph on the Ring of Integers Modulo n," Mathematics and Statistics, Vol.9, No.6, pp. 917-926, 2021. DOI: 10.13189/ms.2021.090607

(b): Magi P M, Sr.Magie Jose, Anjaly Kishore, (2021). Seidel Laplacian and Seidel Signless Laplacian Spectrum of the Zero-divisor Graph on the Ring of Integers Modulo n. Mathematics and Statistics, 9(6), 917-926. DOI: 10.13189/ms.2021.090607

Copyright $@ 2021$ by authors, all rights reserved. Authors agree that this article remains permanently open access under the terms of the Creative Commons Attribution License 4.0 International License

\begin{abstract}
Let $G$ be a simple graph of order $n$ and let $S(G)$ be the Seidel matrix of $G$, defined as $S(G)=\left[s_{i j}\right]$ where $s_{i j}=-1$ if the vertices $v_{i}$ and $v_{j}$ are adjacent and $s_{i j}=1$ if the vertices $v_{i}$ and $v_{j}$ are not adjacent and $s_{i j}=0$ if $i=j$. Let $D_{S}(G)=\operatorname{diag}\left(n-2 d_{1}-1, n-2 d_{2}-1, \ldots, n-2 d_{n}-1\right)$ be the diagonal matrix where $d_{i}$ denotes the degree of the $i^{\text {th }}$ vertex of $G$. The Seidel Laplacian matrix of a graph $G$ is defined as $S L(G)=D_{S}(G)-S(G)$ and the Seidel signless Laplacian matrix of a graph $G$ is defined as $S L^{+}(G)=D_{S}(G)+S(G)$. The zero-divisor graph of a commutative ring $R$, denoted by $\Gamma(R)$, is a simple undirected graph with all non-zero zero-divisors as vertices and two distinct vertices $x, y$ are adjacent if and only if $x y=0$. In this paper, we find the Seidel polynomial and Seidel Laplacian polynomial of the join of two regular graphs using the concept of schur complement and coronal of a square matrix. Also we describe the computation of the Seidel Laplacian and Seidel signless Laplacian eigenvalues of the join of more than two regular graphs, using the well known Fiedler's lemma and apply these results to describe these eigenvalues for the zero-divisor graph on $\mathbb{Z}_{n}$. Further we find the Seidel Laplacian and Seidel signless Laplacian spectrum of the zero-divisor graph of $\mathbb{Z}_{n}$ for some values of $n$, say $n=p^{3}, p^{4}, p q, p^{2} q$, where $p, q$ are distinct primes. We also prove that 0 is a simple Seidel Laplacian eigenvalue of $\Gamma\left(\mathbb{Z}_{n}\right)$, for any $n$.
\end{abstract}

Keywords Seidel Laplacian Matrix, Zero-divisor Graph

\section{Introduction}

Throughout this paper, $G$ denotes a simple, finite, undirected and connected graph. If $G$ has $n$ vertices, then the adjacency matrix, $A(G)=\left[a_{i j}\right]_{n \times n}$ where, $a_{i j}=1$ if $v_{i} \sim v_{j}$ and $a_{i j}=0$ otherwise. Van Lint and Seidel [1] introduced the Seidel matrix of $G$, defined as $S(G)=\left[s_{i j}\right]$ where, $s_{i j}=\left\{\begin{array}{ll}-1 ; & \text { if } v_{i} \sim v_{j} \\ 1 ; & \text { if } v_{i} \not v_{j} \\ 0 ; & \text { otherwise }\end{array}\right.$. Clearly, $S(G)=J-I-2 A(G)$. Also, if $\bar{G}$ denotes the complement of a graph $G$, then $S(\bar{G})=-S(G)$. The Seidel spectrum of $G$ is denoted by $\operatorname{spec}^{S}(G)$. Let $D_{S}(G)=$ $\operatorname{diag}\left(n-2 d_{1}-1, n-2 d_{2}-1, \ldots, n-2 d_{n}-1\right)$ be the diagonal matrix where $d_{i}$ is the degree of the vertex $v_{i}$. The Seidel Laplacian matrix [2] of a graph $G$ is defined as

$$
S L(G)=D_{S}(G)-S(G)
$$


and the Seidel signless Laplacian matrix of a graph $G$ is defined as

$$
S L^{+}(G)=D_{S}(G)+S(G) .
$$

For a complete graph $K_{n}$,

$$
S L\left(K_{n}\right)=J_{n}-n I_{n}
$$

and

For a null graph, $\overline{K_{n}}$,

$$
S L^{+}\left(K_{n}\right)=(2-n) I_{n}-J_{n} .
$$

$$
S L\left(\overline{K_{n}}\right)=n I_{n}-J_{n}
$$

and

$$
S L^{+}\left(\overline{K_{n}}\right)=(n-2) I_{n}+J_{n} .
$$

\section{Basic definitions and notations}

Let $\Phi(G ; x)$ and $\operatorname{Spec}(G)$ denote the characteristic polynomial and the spectrum of $G$ respectively. $\Phi_{S L}(G ; x)$ and $\Phi_{S L^{+}}(G ; x)$ denote the Seidel Laplacian polynomial and Seidel signless Laplacian polynomial of a graph $G$. In this paper, $\mathbf{1}_{n}$ denotes the all-one column vector of order $n \times 1$, and $\phi(n)$ is the number of positive integer less than $n$ and relatively prime to $n$.

Definition 2.1. The join of two graphs $G_{1}$ and $G_{2}$; denoted by $G_{1} \nabla G_{2}$, is obtained by joining each vertex of $G_{1}$ to all the vertices of $G_{2}$.

Definition 2.2. [3] Let the vertices of a graph $G$ be labeled as $1,2,3, \ldots, n$ and let $H_{1}, H_{2}, \ldots, H_{n}$ be a collection of vertex disjoint graphs. The generalized join of $H_{1}, H_{2}, \ldots, H_{n}$ denoted by $G\left[H_{1}, H_{2}, \ldots, H_{n}\right]$, is obtained by replacing each vertex $i$ of $G$ by the graph $H_{i}$ and inserting all or none of the possible edges between $H_{i}$ and $H_{j}$ accordingly if $i$ and $j$ are adjacent in $G$ or not.

Definition 2.3. [4] The sum of the entries of the matrix $(x I-A)^{-1}$ is defined as the coronal of $A$, and is denoted by $\Gamma_{A}(x)$. It can be seen that, $\Gamma_{A}(x)=\left(\mathbf{1}_{n}\right)^{T}$. $(x I-A)^{-1} \cdot \mathbf{1}_{n}$. We note that, if $k$ is the sum of each row, then $\Gamma_{A}(x)=\frac{n}{x-k}$.

I. Beck [5] initiated the idea of zero-divisor graph $G(R)$ of a commutative ring $R$ in connection with some coloring problems and in 1999, Anderson and Livingston [6], modified the definition of zero-divisor graph as a simple graph $\Gamma(R)$ where, only nonzero zero-divisors of $R$ are considered as vertices. The concept of compressed zero-divisor graph; determined by the equivalence classes of the zero-divisors of $R$, was introduced by Mulay [7], with the purpose of simplifying the representation of $\Gamma(R)$ In $[8,9]$, the authors describe the adjacency matrix, eigenvalues and some graph parameters of the zero-divisor graphs $\Gamma\left(\mathbb{Z}_{p^{2} q}\right)$, $\Gamma\left(\mathbb{Z}_{p^{2} q^{2}}\right)$ and $\Gamma\left(\mathbb{Z}_{p^{k}}\right)$. S. Chattopadhyay et.al [10], have explored the combinatorial structure of $\Gamma\left(\mathbb{Z}_{n}\right)$ as the generalized union of its induced subgraphs. The combinatorial properties of the proper divisor graph on $n$ have been investigated in [11]. It is very interesting and challenging that the combinatorial and spectral properties of zero-divisor graphs can be studied in terms of its compressed graph.

\section{Fiedler's Lemma and its generalisation}

The Abel's impossibility theorem implies that the algebraic determination of all the zeroes of a polynomial of degree greater than or equal to five, is impossible in closed form. So, we need resort to the tools of Linear Algebra to explore the eigenvalues of large matrices by means of its sub matrices. In this direction, the famous Fiedler's lemma is very relevant to find the eigenvalues of large symmetric matrices. In [12], H.S.Ramane et.al find the Seidel Laplacian polynomial of the join of two regular graphs. In this section, we find the same in a fairly shorter method, by applying the following lemma and extend the result to the join of more than two regular graphs.

\subsection{Fiedlers's Lemma}

Lemma 3.1. [13] Consider two symmetric matrices $A_{m \times m}$ and $B_{n \times n}$ and let $\left(\alpha_{i}, \mathbf{u}_{i}\right), i=1,2, \ldots, m$ and $\left(\beta_{i}, \mathbf{v}_{i}\right), i=1,2, \ldots, n$, be the eigen pairs of $A$ and $B$ respectively, where $\left\|\mathbf{u}_{1}\right\|=1=\left\|\mathbf{v}_{1}\right\|$. For any arbitrary constant $\rho$, let the matrix $\widehat{C}=\left[\begin{array}{cc}\alpha_{1} & \rho \\ \rho & \beta_{1}\end{array}\right]$ has eigenvalues $\gamma_{1}, \gamma_{2}$. Then, $C=\left[\begin{array}{cc}A & \rho \mathbf{u}_{1} \mathbf{v}_{1}^{T} \\ \rho \mathbf{v}_{1} \mathbf{u}_{1}^{T} & B\end{array}\right]$ has eigenvalues $\alpha_{2}, \ldots, \alpha_{m}, \beta_{2}, \ldots, \beta_{n}, \gamma_{1}, \gamma_{2}$.

Lemma 3.2. [14] If $G$ is a $k$-regular graph of order $n$, and $k, \lambda_{2}, \ldots, \lambda_{n}$ are the adjacency eigenvalues of $G$, then $\theta_{1}=n-2 k-1, \theta_{2}=-1-2 \lambda_{2}, \theta_{3}=-1-2 \lambda_{3}, \ldots, \theta_{n}=-1-2 \lambda_{n}$ are the Seidel eigenvalues of $G$. 
Theorem 3.1. Let $G_{i}$ be $r_{i}$ regular of order $n_{i}$, for $i=1,2$. Then the Seidel Laplacian polynomial of $G_{1} \nabla G_{2}$ is given by

$$
\Phi_{S L}\left(G_{1} \nabla G_{2} ; x\right)=\frac{x\left(x+n_{1}+n_{2}\right)}{\left(x+n_{1}\right)\left(x+n_{2}\right)} \cdot \Phi_{S L}\left(G_{1} ; x+n_{2}\right) \cdot \Phi_{S L}\left(G_{2} ; x+n_{1}\right) .
$$

Proof. Let $S\left(G_{1}\right)$ and $S\left(G_{2}\right)$ denote the Seidel adjacency matrices of $G_{1}$ and $G_{2}$ respectively. Then, the Seidel Laplacian matrices of $G_{1}$ and $G_{2}$ are given as follows.

$$
\begin{aligned}
& S L\left(G_{1}\right)=\left(n_{1}-2 r_{1}-1\right) I_{n_{1}}-S\left(G_{1}\right), \\
& S L\left(G_{2}\right)=\left(n_{2}-2 r_{2}-1\right) I_{n_{2}}-S\left(G_{2}\right) .
\end{aligned}
$$

Since $G_{1}$ is regular, by Lemma 3.2, $n_{1}-2 r_{1}-1$ is an eigenvalue of $S\left(G_{1}\right)$ with eigenvector $\mathbf{1}_{n_{1}}$. Also, 1 is an eigenvalue of $I_{n_{1}}$ with eigenvector $\mathbf{1}_{n_{1}}$. Thus, $n_{1}-2 r_{1}-1$ is an eigenvalue of both $S\left(G_{1}\right)$ and $\left(n_{1}-2 r_{1}-1\right) I_{n_{1}}$ with associated eigenvector $\mathbf{1}_{n_{1}}$, which means that, 0 is an eigenvalue of $S L\left(G_{1}\right)$ with eigenvector $\mathbf{1}_{n_{1}}$. Similarly, 0 is an eigenvalue of $S L(G)$ with corresponding eigenvector $\mathbf{1}_{n_{2}}$.

Clearly,

$$
\begin{aligned}
S L\left(G_{1} \nabla G_{2}\right) & =\left[\begin{array}{cc}
\left(n_{1}+n_{2}-2\left(r_{1}+n_{2}\right)-1\right) I_{n_{1}}-S_{1} & J_{n_{1} \times n_{2}} \\
J_{n_{1} \times n_{2}}^{T} & \left(n_{1}+n_{2}-2\left(r_{2}+n_{1}\right)-1\right) I_{n_{2}}-S_{2}
\end{array}\right] \\
& =\left[\begin{array}{cc}
S L\left(G_{1}\right)-n_{2} I_{n_{1}} & J_{n_{1} \times n_{2}} \\
J_{n_{1} \times n_{2}}^{T} & S L\left(G_{2}\right)-n_{1} I_{n_{2}}
\end{array}\right] .
\end{aligned}
$$

Taking $A=S L\left(G_{1}\right)-n_{2} I_{n_{1}}, B=S L\left(G_{2}\right)-n_{1} I_{n_{2}} \alpha_{1}=-n_{2}, \beta_{1}=-n_{1}, \mathbf{u}_{1}=\frac{1}{\sqrt{n_{1}}} \mathbf{1}_{n_{1}}, \mathbf{u}_{2}=\frac{1}{\sqrt{n_{2}}} \mathbf{1}_{n_{2}}, \rho=\sqrt{n_{1} n_{2}}$ and applying Fiedler's Lemma, it can be seen that

$$
\operatorname{spec}\left(S L\left(G_{1} \nabla G_{2}\right)\right)=\operatorname{spec}\left(S L\left(G_{1}\right)-n_{2} I_{n_{1}}\right) \backslash\left\{-n_{2}\right\} \cup \operatorname{spec}\left(S L\left(G_{2}\right)-n_{1} I_{n_{2}}\right) \backslash\left\{-n_{1}\right\} \cup \operatorname{spec} \hat{C},
$$

where the matrix $\hat{C}=\left[\begin{array}{cc}-n_{2} & \sqrt{n_{1} n_{2}} \\ \sqrt{n_{1} n_{2}} & -n_{1}\end{array}\right]$. Clearly, spec $\hat{C}=\left\{0,-n_{1}-n_{2}\right\}$. Thus,

$$
\Phi_{S L}\left(G_{1} \nabla G_{2} ; x\right)=\frac{x\left(x+n_{1}+n_{2}\right)}{\left(x+n_{1}\right)\left(x+n_{2}\right)} \cdot \Phi_{S L}\left(G_{1} ; x+n_{2}\right) \cdot \Phi_{S L}\left(G_{2} ; x+n_{1}\right) \text {. }
$$

\subsection{Generalization of Fiedler's Lemma}

Let $M_{j}$ be an $m_{j} \times m_{j}$ symmetric matrix, with corresponding eigenpairs $\left(\alpha_{r j}, \mathbf{u}_{r j}\right), 1 \leqslant r \leqslant m_{j}$, where $j \in\{1,2, \ldots, k\}$. Also, for $q \in\{1,2, \ldots, k-1\}$ and $l \in\{q+1, \ldots, k\}$, let $\rho_{q, l}$ be arbitrary real numbers. Let $\hat{\alpha}$ be the $k-$ tuple

$$
\hat{\alpha}=\left(\alpha_{i_{1}, 1}, \ldots, \alpha_{i_{k}, k}\right)
$$

where each $\alpha_{i_{j}, j}$ is selected from the collection of $\left\{\alpha_{1, j}, \ldots, \alpha_{m_{j}, j}\right\}$ where $j \in\{1,2, \ldots, k\}$. Taking $\hat{\rho}$ as a $\frac{k(k-1)}{2}$ - tuple of real numbers,

$$
\hat{\rho}=\left(\rho_{1,2}, \rho_{1,3}, \ldots, \rho_{1, k}, \rho_{2,3}, \ldots, \rho_{2, k}, \ldots, \rho_{k-1, k}\right)
$$

consider the symmetric matrices

$C_{\widehat{\alpha}}(\hat{\rho})=$

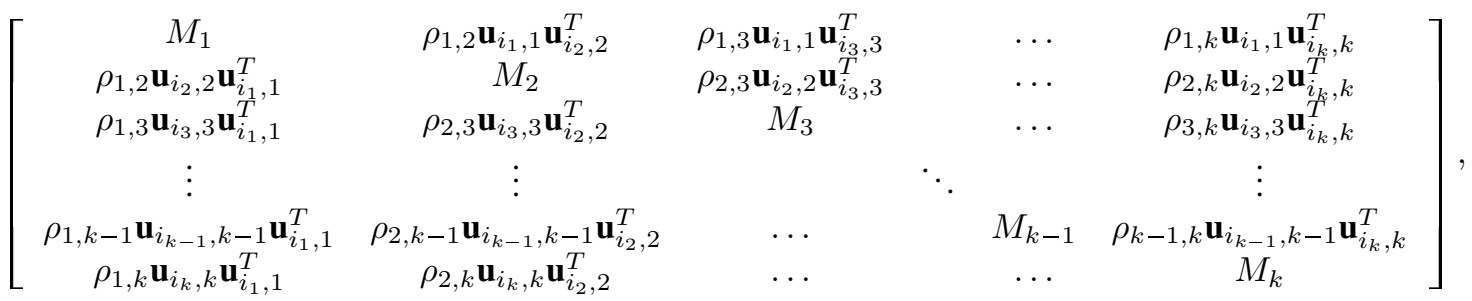

$$
\begin{aligned}
& \widetilde{C}_{\widehat{\alpha}}(\hat{\rho})=\left[\begin{array}{ccccc}
\alpha_{i_{1}, 1} & \rho_{1,2} & \ldots & \rho_{1, k-1} & \rho_{1, k} \\
\rho_{1,2} & \alpha_{i_{2}, 2} & \ldots & \rho_{2, k-1} & \rho_{2, k} \\
\vdots & \vdots & \ddots & \vdots & \vdots \\
\rho_{1, k-1} & \rho_{2, k-1} & \ldots & \alpha_{i_{k-1}, k-1} & \rho_{k-1, k} \\
\rho_{1, k} & \rho_{2, k} & \ldots & \rho_{k-1, k} & \alpha_{i_{k}, k}
\end{array}\right]_{k \times k}
\end{aligned}
$$


920 Seidel Laplacian and Seidel Signless Laplacian Spectrum of the Zero-divisor Graph on the Ring of Integers Modulo $n$

Theorem 3.2. [15] For $j \in\{1,2, \ldots, k\}$, let $M_{j}, \hat{\rho}, \hat{\alpha}$ be as defined (1) and (2). Suppose that for each $j$, the system of eigen vectors $\left\{\mathbf{u}_{r j}, r \in I_{j}\right\}$ is orthonormal. Then the matrix $C_{\widehat{\alpha}}(\hat{\rho})$ in (3) has the multi set of eigenvalues $\left(\bigcup_{j=1}^{k}\left\{\alpha_{1, j}, \ldots, \alpha_{m_{j}, j}\right\} \backslash\left\{\alpha_{i_{j}, j}\right\}\right) \cup$ $\left\{\gamma_{1}, \ldots, \gamma_{k}\right\}$, where $\gamma_{1}, \gamma_{2}, \ldots, \gamma_{k}$ are eigenvalues of the matrix $\widetilde{C}_{\widehat{\alpha}}(\hat{\rho})$ in (4).

The authors D.M. Cardoso et al [ 15] and Magi.P.M. et al [16] have used the above result to find distance related spectrum of the generalized join of graphs.

\section{Seidel Laplacian spectrum of the joined union of regular graphs}

Consider $G\left[H_{1}, H_{2}, \ldots, H_{k}\right]$, where the vertices of $G$ are labeled as $1,2, \ldots, k$, and the Seidel matrix $S(G)=\left[s_{i j}\right]$. Let $H_{j}$ be $r_{j}$-regular and $\left|V\left(H_{j}\right)\right|=n_{j}$, for every $j=1,2, \ldots, k$. Let $S\left(H_{j}\right)$ and $S L\left(H_{j}\right)$ denote the Seidel matrix and Seidel Laplacian matrix of $H_{j}, j=1,2, \ldots, k$. The degree of each vertex of $H_{j}$, in the joined graph $G\left[H_{1}, H_{2}, \ldots, H_{k}\right]$ is $r_{j}+\sum_{i \sim j} n_{i}$. Hence, if $S_{j}$ denotes the $j^{\text {th }}$ diagonal block in the Seidel Laplacian matrix of $G\left[H_{1}, H_{2}, \ldots, H_{k}\right]$, then,

$$
\begin{aligned}
S_{j} & =\left(\sum_{i=1}^{k} n_{i}-2\left(r_{j}+\sum_{j \sim i} n_{i}\right)-1\right) I_{n_{j}}-S\left(H_{j}\right) \\
& =\left(n_{j}-2 r_{j}-1\right) I_{n_{j}}-S\left(H_{j}\right)+\left(\sum_{j \neq i} n_{i}-\sum_{j \sim i} n_{i}\right) I_{n_{j}} \\
& =S L\left(H_{j}\right)+\left(\sum_{i=1}^{k} s_{i j} n_{i}\right) I_{n_{j}} \\
& =S L\left(H_{j}\right)+\tau_{j} I_{n_{j}} .
\end{aligned}
$$

where $\tau_{j}=\sum_{i=1}^{k} s_{i j} n_{i}$.

Thus, we see that, the Seidel Laplacian matrix of the $G$ - union of $H_{1}, H_{2}, \ldots, H_{k}$ is given by

$$
S L\left(G\left[H_{1}, H_{2}, \ldots, H_{k}\right]\right)=\left[\begin{array}{cccc}
S L\left(H_{1}\right)+\tau_{1} I_{n_{1}} & -s_{1,2} J_{n_{1} \times n_{2}} & \ldots & -s_{1, k} J_{n_{1} \times n_{k}} \\
-s_{1,2} J_{n_{1} \times n_{2}}^{T} & S L\left(H_{2}\right)+\tau_{2} I_{n_{2}} & \ldots & -s_{2, k} J_{n_{2} \times n_{k}} \\
\vdots & & \ddots & \vdots \\
-s_{1, k} J_{n_{1} \times n_{k}}^{T} & -s_{2, k} J_{n_{2} \times n_{k}}^{T} & \ldots & S L\left(H_{k}\right)+\tau_{k} I_{n_{k}}
\end{array}\right]
$$

where $\tau_{j}=\sum_{i=1}^{k} s_{i j} n_{i}$.

Remark 4.1. Since for a $k$-regular graph $G$ of order $n, S L(G)=(n-2 k-1) I_{n}-S(G)$, from Lemma 3.2 it follows that, $S L(G)$ has an eigenvalue 0 with multiplicity at least 1 . For example, the Seidel Laplacian spectrum of the cycle $C_{4}$, which is 2 regular is $\operatorname{spec}^{S L}\left(C_{4}\right)=\left\{\begin{array}{cc}0 & -4 \\ 3 & 1\end{array}\right\}$. However, the Seidel Laplacian matrix of complete graphs and null graphs (complement of complete graphs) has an eigenvalue 0 with multiplicity 1.

Theorem 4.1. Consider $G\left[H_{1}, H_{2}, \ldots, H_{k}\right]$ and $S=\left[s_{i j}\right]_{k \times k}$ is the Seidel matrix of $G$ and $H_{j}$ is $r_{j}$-regular and $\left|V\left(H_{j}\right)\right|=n_{j}$, for every $j=1,2, \ldots, k$. Let $\left\{\sigma_{j 1}^{S L}=0, \sigma_{j 2}^{S L}, \ldots, \sigma_{j n_{j}}^{S L}\right\}$ be the Seidel Laplacian eigenvalues of $H_{j}$, for $j=1,2, \ldots, k$. Then,

$$
\operatorname{spec}^{S L}\left(G\left[H_{1}, H_{2}, \ldots, H_{n}\right]\right)=\left(\bigcup_{j=1}^{k} \bigcup_{i=2}^{n_{j}}\left(\sigma_{j i}^{S L}+\tau_{j}\right)\right) \cup \operatorname{spec}\left(T_{S L}(G)\right),
$$

where $\tau_{j}=\sum_{i=1}^{k} s_{i j} n_{i}$ and

$$
T_{S L}(G)=\left[\begin{array}{cccc}
\tau_{1} & -s_{1,2} n_{2} & \ldots & -s_{1, k} n_{k} \\
-s_{1,2} n_{1} & \tau_{2} & \ldots & -s_{2, k} n_{k} \\
\vdots & \vdots & \ddots & \vdots \\
-s_{1, k} n_{1} & -s_{2, k} n_{2} & \cdots & \tau_{k}
\end{array}\right]
$$

Proof. Since $H_{j}$ is regular, 0 is a Seidel Laplacian eigenvalue of $H_{j}$ with eigenvector $\mathbf{1}_{n_{j}}$, for every $j$. Thus from (5), it is evident that each of the diagonal blocks $S L\left(H_{j}\right)+\tau_{j} I_{n_{j}}$ is a symmetric matrix which has $\tau_{j}$ as an eigenvalue with eigenvector $\mathbf{1}_{n_{j}}$, $j=1,2, \ldots, k$. 
As in (3), taking

$$
M_{j}=S L\left(H_{j}\right)+\tau_{j} I_{n_{j}}, \quad\left(\alpha_{i_{j}, j}, \mathbf{u}_{i_{j}, j}\right)=\left(\tau_{j}, \frac{1}{\sqrt{n_{j}}} \mathbf{1}_{n_{j}}\right)
$$

and the real numbers

$$
\rho_{l, q}=-s_{l q} \sqrt{n_{l} n_{q}}
$$

for $l \in\{1,2, \ldots, k-1\}, q \in\{l+1, \ldots, k\}$, and applying Theorem 3.2, we find that,

$$
\operatorname{spec}^{S L}\left(G\left[H_{1}, H_{2}, \ldots, H_{n}\right]\right)=\left(\bigcup_{j=1}^{k} \operatorname{spec}\left(S L\left(H_{j}\right)+\tau_{j} I_{n_{j}}\right) \backslash\left\{\tau_{j}\right\}\right) \cup \operatorname{spec}((\widetilde{S}))
$$

where $\tau_{j}=\sum_{i=1}^{k} s_{i j} n_{i}$ and $\widetilde{S}=\left[\begin{array}{cccc}\tau_{1} & \rho_{1,2} & \ldots & \rho_{1, k} \\ \rho_{1,2} & \tau_{2} & \ldots & \rho_{2, k} \\ \vdots & \vdots & \ddots & \vdots \\ \rho_{1, k} & \rho_{2, k} & \ldots & \tau_{k}\end{array}\right]$.

Obviously, $\rho_{l, q}=-s_{l q} \sqrt{n_{l} n_{q}}=\left\{\begin{array}{ll}\sqrt{n_{l} n_{q}} & \text { if } l q \in \mathrm{E}(\mathrm{G}) \\ -\sqrt{n_{l} n_{q}} & \text { if } l q \notin \mathrm{E}(\mathrm{G})\end{array} \quad\right.$ for $l \in\{1,2, \ldots, k-1\}, q \in\{l+1, \ldots, k\}$.

Assign the weight $n_{j}=\left|V\left(H_{j}\right)\right|$ to the vertex $j$ of $G$ for $j=1,2, \ldots, k$ and consider the matrix $W$ which is a diagonal matrix of vertex weights,

$$
W=\left[\begin{array}{cccc}
n_{1} & 0 & \ldots & 0 \\
0 & n_{2} & \ldots & 0 \\
\vdots & & \ddots & \vdots \\
0 & \ldots & \cdots & n_{k}
\end{array}\right]
$$

Let $T_{S L}(G)$ be the combinatorial ( vertex weighted ) Seidel Laplacian matrix of $G$, given by

$$
T_{S L}(G)=\left[\begin{array}{cccc}
\tau_{1} & -s_{1,2} n_{2} & \ldots & -s_{1, k} n_{k} \\
-s_{1,2} n_{1} & \tau_{2} & \ldots & -s_{2, k} n_{k} \\
\vdots & \vdots & \ddots & \vdots \\
-s_{1, k} n_{1} & -s_{2, k} n_{2} & \ldots & \tau_{k}
\end{array}\right]
$$

The vertex weighted combinatorial Laplacian matrix of graphs can be seen in [17]. It is easy to verify that $T_{S L}(G)=W^{-\frac{1}{2}} \widetilde{S} W^{\frac{1}{2}}$. Thus $\widetilde{S}$ and $T_{S L}(G)$ are similar and hence $\operatorname{spec}(\widetilde{S})=\operatorname{spec}\left(T_{S L}(G)\right)$. Hence from $(6)$,

$$
\left.\operatorname{spec}^{S L}\left(G\left[H_{1}, H_{2}, \ldots, H_{n}\right]\right)=\left(\bigcup_{j=1}^{k} \bigcup_{i=2}^{n_{j}}\left(\sigma_{j i}^{S L}+\tau_{j}\right)\right) \cup \operatorname{spec}\left(T_{S L}(G)\right)\right) .
$$

\section{The zero-divisor graph $\Gamma\left(\mathbb{Z}_{n}\right)$}

The commutative ring $\mathbb{Z}_{p}$ is an integral domain for any prime $p$ and so it is quite trivial to study its zero-divisor graph. Hence in the following sessions, it is assumed that $n$ is not a prime. It is very relevant to note that $\Gamma\left(\mathbb{Z}_{p^{2}}\right)$ is a complete graph on $p-1$ vertices. Also $\Gamma\left(\mathbb{Z}_{8}\right), \Gamma\left(\mathbb{Z}_{p q}\right)$ are complete bipartite graphs. Using elementary number theory, the number of non zero zero-divisors is calculated to be $n-\phi(n)-1$ [9].

For distinct primes $p_{1}, p_{2}, \ldots, p_{r}$, let $n=p_{1}^{n_{1}} \cdot p_{2}^{n_{2}} \cdots p_{r}^{n_{r}}$ be the canonical decomposition of $n$. A proper divisor of $n$ is a positive divisor $d$ such that $d \mid n, 1<d<n$. Let $p(n)$ denote the number of proper divisors of $n$. Then, $p(n)=\sigma_{0}(n)-2$, where $\sigma_{k}(n)$ is the sum of $k$ powers of all divisors of $n$, including $n$ and 1 .

Let $\mathbf{S}(d)=\left\{k \in \mathbb{Z}_{n}: \operatorname{gcd}(k, n)=d\right\}$. Then $\left\{\mathbf{S}\left(d_{1}\right), \mathbf{S}\left(d_{2}\right), \ldots, \mathbf{S}\left(d_{p(n)}\right)\right\}$ forms an equitable partition of $V\left(\Gamma\left(\mathbb{Z}_{n}\right)\right)$ Also, $\left|\mathbf{S}\left(d_{i}\right)\right|=\phi\left(\frac{n}{d_{i}}\right)$, for every $i=1,2, \ldots p(n)$. The following Lemmas are inevitable in the analysis of $\Gamma\left(\mathbb{Z}_{n}\right)$.

Lemma 5.1. [10] Let $\Gamma\left(\mathbf{S}\left(d_{i}\right)\right)$ denote the subgraph of $\Gamma\left(\mathbb{Z}_{n}\right)$, induced by $\mathbf{S}\left(d_{i}\right)$ for $i=1,2, \ldots, p(n)$. Then,

$\Gamma\left(\mathbf{S}\left(d_{i}\right)\right)=\left\{\begin{array}{ll}\bar{K}_{\phi\left(\frac{n}{d_{i}}\right)} & \text { if } n \nmid d_{i}^{2} \\ K_{\phi\left(\frac{n}{d_{i}}\right)} & \text { if } n \mid d_{i}^{2}\end{array}\right.$. 
Lemma 5.2. [10] $\Gamma\left(\mathbb{Z}_{n}\right)=\Upsilon_{n}\left[\Gamma\left(\mathbf{S}\left(d_{1}\right)\right), \Gamma\left(\mathbf{S}\left(d_{2}\right)\right), \ldots, \Gamma\left(\mathbf{S}\left(d_{p(n)}\right)\right)\right]$

The subgraph $\Gamma\left(\mathbf{S}\left(d_{i}\right)\right)$ is regular for each $i=1,2, \ldots, p(n)$. For example, in $\Gamma\left(\mathbb{Z}_{p^{3}}\right), \mathbf{S}(p)$ induces $\bar{K}_{p(p-1)}$ and $\mathbf{S}\left(p^{2}\right)$ induces $K_{p-1}$. In $\Gamma\left(\mathbb{Z}_{p^{2} q}\right), \mathbf{S}(p), \mathbf{S}(q), \mathbf{S}\left(p^{2}\right)$ induce $\bar{K}_{(p-1)(q-1)}, \bar{K}_{p(p-1)}, \bar{K}_{q-1}$ respectively whereas $\mathbf{S}(p q)$ induces $K_{p-1}$.

The proper divisor graph, denoted by $\Upsilon_{n}$ plays a vital role in the analysis of $\Gamma\left(\mathbb{Z}_{n}\right)$, the vertices of which are labeled as $d_{1}, d_{2}, \ldots, d_{p(n)}$. See[11]. Also $d_{i} \sim d_{j}$ in $\Upsilon_{n}$ if and only if $n$ divides the product $d_{i} d_{j}$. [10].

\subsection{Seidel Laplacian spectrum of $\Gamma\left(\mathbb{Z}_{n}\right)$}

Theorem 5.1. If $d_{1}, d_{2}, \ldots, d_{p(n)}$ are the proper divisors of $n$, then the Seidel Laplacian matrix of $\Gamma\left(\mathbb{Z}_{n}\right)$ is given by

$$
S L\left(\Gamma\left(\mathbb{Z}_{n}\right)\right)=\left[\begin{array}{cccc}
S_{1} & -s_{1,2} J_{\phi\left(\frac{n}{d_{1}}\right) \times \phi\left(\frac{n}{d_{2}}\right)} & \cdots & -s_{1, p(n)} J_{\phi\left(\frac{n}{d_{1}}\right) \times \phi\left(\frac{n}{d_{p(n)}}\right)} \\
-s_{1,2} J_{\phi\left(\frac{n}{d_{1}}\right) \times \phi\left(\frac{n}{d_{2}}\right)}^{T} & S_{2} & \ldots & -s_{2, p(n)} J_{\phi\left(\frac{n}{d_{2}}\right) \times \phi\left(\frac{n}{d_{p(n)}}\right)} \\
\vdots & \vdots & \ddots & \vdots \\
-s_{1, p(n)} J_{\phi\left(\frac{n}{d_{1}}\right) \times \phi\left(\frac{n}{d_{p(n)}}\right)}^{T} & -s_{2, p(n)} J_{\phi\left(\frac{n}{d_{2}}\right) \times \phi\left(\frac{n}{d_{p(n)}}\right)}^{T} & \cdots & S_{p(n)}
\end{array}\right]
$$

where,

$$
S_{j}= \begin{cases}\left(\phi\left(\frac{n}{d_{j}}\right)+\sum_{i=1}^{p(n)} s_{i j} \phi\left(\frac{n}{d_{i}}\right)\right) I_{\phi\left(\frac{n}{d_{j}}\right)}-J_{\phi\left(\frac{n}{d_{j}}\right.} & \text { if } n \nmid d_{j}^{2} \\ J_{\phi}\left(\frac{n}{d_{j}}\right)+\left(\sum_{i=1}^{p(n)} s_{i j} \phi\left(\frac{n}{d_{i}}\right)-\phi\left(\frac{n}{d_{j}}\right)\right) I_{\phi\left(\frac{n}{d_{j}}\right)} & \text { if } n \mid d_{j}^{2}\end{cases}
$$

and

$$
s_{i j}= \begin{cases}-1 & \text { if } n \mid d_{i} d_{j} \\ 1 & \text { if } n \nmid d_{i} d_{j}\end{cases}
$$

Proof. In the proper divisor graph $\Upsilon_{n}$, the vertices $d_{i}$ and $d_{j}$ are adjacent if and only if $n \mid d_{i} d_{j}$. Thus if $S=\left[s_{i j}\right]_{p(n) \times p(n)}$ denotes the Seidel adjacency matrix of $\Upsilon_{n}$, then

$$
s_{i j}= \begin{cases}-1 & \text { if } n \mid d_{i} d_{j} \\ 1 & \text { if } n \nmid d_{i} d_{j}\end{cases}
$$

By Lemma 5.2, $\Gamma\left(\mathbb{Z}_{n}\right)$ is the $\Upsilon_{n}$ - join of $\Gamma\left(\mathbf{S}\left(d_{1}\right)\right), \Gamma\left(\mathbf{S}\left(d_{2}\right)\right), \ldots, \Gamma\left(\mathbf{S}\left(d_{p(n)}\right)\right)$. The induced subgraphs $\Gamma\left(\mathbf{S}\left(d_{j}\right)\right)$ are either a complete graph or a null graph on $\phi\left(\frac{n}{d_{j}}\right)$ vertices.

By Lemma 5.1,

$$
\Gamma\left(\mathbf{S}\left(d_{j}\right)\right)=\left\{\begin{array}{ll}
\bar{K}_{\phi\left(\frac{n}{d_{j}}\right)} & \text { if } n \nmid d_{j}^{2} \\
K_{\phi\left(\frac{n}{d_{j}}\right)} & \text { if } n \mid d_{j}^{2}
\end{array} .\right.
$$

The Seidel Laplacian matrix of $K_{n}$ and $\overline{K_{n}}$ are given by

$$
\begin{aligned}
& S L\left(K_{n}\right)=J_{n}-n I_{n}, \\
& S L\left(\overline{K_{n}}\right)=n I_{n}-J_{n} .
\end{aligned}
$$

Thus, if $S_{j}$ denotes the $j^{\text {th }}$ diagonal block in the Seidel Laplacian matrix of $\Gamma\left(\mathbb{Z}_{n}\right)$, which corresponds to the vertices of $\Gamma\left(\mathbf{S}\left(d_{j}\right)\right)$, then, $S_{j}=S L\left(\Gamma\left(\mathbf{S}\left(d_{j}\right)\right)\right)+\tau_{j} I_{\phi\left(\frac{n}{d_{j}}\right)} \quad$ where, $\tau_{j}=\sum_{i=1}^{p(n)} s_{i j} \phi\left(\frac{n}{d_{i}}\right)$, from (5). Hence,

$$
S_{j}=\left\{\begin{array}{ll}
\phi\left(\frac{n}{d_{j}}\right) I_{\phi\left(\frac{n}{d_{j}}\right)}-J_{\phi\left(\frac{n}{d_{j}}\right)}+\tau_{j} I_{\phi\left(\frac{n}{d_{j}}\right)} & \text { if } n \nmid d_{j}^{2} \\
J_{\phi\left(\frac{n}{d_{j}}\right)}-\phi\left(\frac{n}{d_{j}}\right) I_{\phi\left(\frac{n}{d_{j}}\right)}+\tau_{j} I_{\phi\left(\frac{n}{d_{j}}\right)} & \text { if } n / d_{j}^{2}
\end{array} .\right.
$$

Thus, the result follows from (5), taking $G=\Upsilon_{n}$ and $H_{j}=\Gamma\left(\mathbf{S}\left(d_{j}\right)\right)$.

Applying Theorem 4.1 and Theorem 5.1, we determine the Seidel Laplacian spectrum of $\Gamma\left(\mathbb{Z}_{n}\right)$ in the following corollary.

Corollary 5.1. Let $d_{1}, d_{2}, \ldots, d_{p(n)}$ be the proper divisors of $n$. Then, $\Gamma\left(\mathbb{Z}_{n}\right)$ has Seidel Laplacian eigenvalues $\phi\left(\frac{n}{d_{j}}\right)+$ $\sum_{i=1}^{p(n)} s_{i j} \phi\left(\frac{n}{d_{i}}\right)$ with multiplicity $\phi\left(\frac{n}{d_{j}}\right)-1$ corresponding to the divisors $d_{j}$ such that $n \nmid d_{j}^{2}$ and $\sum_{i=1}^{p(n)} s_{i j} \phi\left(\frac{n}{d_{i}}\right)-\phi\left(\frac{n}{d_{j}}\right)$ 
with multiplicity $\phi\left(\frac{n}{d_{j}}\right)-1$ corresponding to the divisors $d_{j}$ such that $n \mid d_{j}^{2}$ and the remaining Seidel Laplacian eigenvalues are the eigenvalues of

$$
T_{S L}(G)=\left[\begin{array}{cccc}
\tau_{1} & -s_{1,2} \phi\left(\frac{n}{d_{2}}\right) & \ldots & -s_{1, p(n)} \phi\left(\frac{n}{d_{p(n)}}\right) \\
-s_{1,2} \phi\left(\frac{n}{d_{1}}\right) & \tau_{2} & \ldots & -s_{2, p(n)} \phi\left(\frac{n}{d_{p(n)}}\right) \\
\vdots & \vdots & \ddots & \vdots \\
-s_{1, p(n)} \phi\left(\frac{n}{d_{1}}\right) & -s_{2, k} \phi\left(\frac{n}{d_{2}}\right) & \ldots & \tau_{p(n)}
\end{array}\right]
$$

where $\tau_{j}=\sum_{i=1}^{p(n)} s_{i j} \phi\left(\frac{n}{d_{i}}\right), j=1,2, \ldots, p(n)$.

Definition 5.1. [18] A matrix $\left[a_{i j}\right]$ is strictly diagonally dominant if in every row of the matrix, the magnitude of the diagonal entry is strictly greater than the sum of the magnitudes of all other non-diagonal entries, that is if, $\quad\left|a_{i i}\right|>\sum_{j \neq i}\left|a_{i j}\right|$, for all $i$.

Theorem 5.2. (Levy- Desplanques, Theorem [18]) A strictly diagonally dominant matrix is non-singular.

Theorem 5.3. 0 ia a simple Seidel Laplacian eigenvalue of $\Gamma\left(\mathbb{Z}_{n}\right)$, for any $n$.

Proof. First, we show that 0 is an eigenvalue of the matrix $T_{S L}(G)$ of multiplicity 1 . For this, we prove that $T_{S L}(G)$ is singular with nullity 1 . Arrange the proper divisors of $n$ in the ascending order, $d_{1}<d_{2}<\ldots<d_{p(n)}$. It is obvious that, $\phi\left(\frac{n}{d_{1}}\right)>$ $\phi\left(\frac{n}{d_{i}}\right), i=2,3, \ldots, p(n)$. We note that $T_{S L}(G)$ is a square matrix of size $p(n)$. Since, $\tau_{j}=\sum_{i=1}^{p(n)} s_{i j} \phi\left(\frac{n}{d_{i}}\right)$,

$$
\tau_{j}+\sum_{i \neq j}-s_{i j} \phi\left(\frac{n}{d_{i}}\right)=0
$$

Hence it follows from (9), that the sum of each row of $T_{S L}(G)$ is zero. The first column of $T_{S L}(G)$ can be transformed to the zero column on adding $2^{n d}, 3^{r d}, \ldots, p(n)^{t h}$ columns to it. Hence $T_{S L}(G)$ is singular which implies that 0 is an eigenvalue of $T_{S L}(G)$. To prove that the multiplicity is 1 , it suffices to prove that the rank of $T_{S L}(G)$ is $p(n)-1$. For this, consider the matrix $T$ obtained from $T_{S L}(G)$ by deleting the first row and first column of $T_{S L}(G)$. Since

$$
s_{i j}= \pm 1, \quad \phi\left(\frac{n}{d_{1}}\right)>\phi\left(\frac{n}{d_{i}}\right), i=2,3, \ldots, p(n) \Rightarrow\left|\tau_{j}\right|>\sum_{i \neq j, i \neq 1}\left|s_{i, j} \phi\left(\frac{n}{d_{i}}\right)\right| .
$$

Thus, $T$ is strictly diagonally dominant. For example, consider the first row of $T$, say

$\left[\tau_{2},-s_{2,3} \phi\left(\frac{n}{d_{3}}\right),-s_{2,4} \phi\left(\frac{n}{d_{4}}\right), \ldots,-s_{2, p(n)} \phi\left(\frac{n}{d_{p(n)}}\right)\right]$. Since, $\tau_{2}=s_{1,2} \phi\left(\frac{n}{d_{1}}\right)+s_{2,3} \phi\left(\frac{n}{d_{3}}\right)+\ldots .+s_{2, p(n)} \phi\left(\frac{n}{d_{p(n)}}\right)$, it follows that, $\left|\tau_{2}\right|>\left|s_{2,3} \phi\left(\frac{n}{d_{3}}\right)\right|+\ldots+\left|s_{2, p(n)} \phi\left(\frac{n}{d_{p(n)}}\right)\right|$.

Hence by Theorem 5.2, $T$ is non-singular, and hence the rank of $T_{S L}(G)$ is $p(n)-1$.

By Corollary 5.1, the remaining Seidel eigenvalues of $\Gamma\left(\mathbb{Z}_{n}\right)$ are $\phi\left(\frac{n}{d_{j}}\right)+\sum_{i=1}^{p(n)} s_{i j} \phi\left(\frac{n}{d_{i}}\right)$ and $\sum_{i=1}^{p(n)} s_{i j} \phi\left(\frac{n}{d_{i}}\right)-\phi\left(\frac{n}{d_{j}}\right)$, neither of which is zero. This proves the theorem.

Theorem 5.4. For distinct primes $p$ and $q, p<q$, the Seidel Laplacian spectrum of $\Gamma\left(\mathbb{Z}_{p q}\right)$ is given by

$$
\operatorname{Spec}^{S L}\left(\Gamma\left(\mathbb{Z}_{p q}\right)\right)=\left\{\begin{array}{cccc}
0 & -(p+q-2) & p-q & q-p \\
1 & 1 & p-2 & q-2
\end{array}\right\}
$$

Proof. The proper divisors of $p q$ are $p$ and $q$. By Lemma 5.1 and Lemma 5.2, the zero-divisor graph $\Gamma\left(\mathbb{Z}_{p q}\right)$ is the join of $\Gamma(\mathbf{S}(p))$ and $\Gamma(\mathbf{S}(q))$, where $\Gamma(\mathbf{S}(p))=\bar{K}_{q-1}$ and $\Gamma(\mathbf{S}(q))=\bar{K}_{p-1}$. That is

$$
\Gamma\left(\mathbb{Z}_{p q}\right)=\bar{K}_{q-1} \nabla \bar{K}_{p-1}
$$

Clearly spec ${ }^{S L}\left(\bar{K}_{q-1}\right)=\left\{\begin{array}{ll}0 & q-1 \\ 1 & q-2\end{array}\right\}$ and $\operatorname{spec}^{S L}\left(\bar{K}_{p-1}\right)=\left\{\begin{array}{ll}0 & p-1 \\ 1 & p-2\end{array}\right\}$. And the result follows from Theorem 3.1.

Theorem 5.5. For any prime $p, \quad$ the Seidel Laplacian spectrum of $\Gamma\left(\mathbb{Z}_{p^{3}}\right)$ is

$$
\operatorname{spec}^{S L}\left(\Gamma\left(\mathbb{Z}_{p^{3}}\right)\right)=\left\{\begin{array}{ccc}
0 & 1-p^{2} & p^{2}-2 p+1 \\
1 & p-1 & p^{2}-p-1
\end{array}\right\}
$$

Proof. The proper divisors of $p^{3}$ are $p$ and $p^{2}$. By lemma 5.1 it can be seen that the subgraphs of $\Gamma\left(\mathbb{Z}_{p^{3}}\right)$, induced by $\mathbf{S}(p)$ and $\mathbf{S}\left(p^{2}\right)$ are $\bar{K}_{p(p-1)}$ and $K_{p-1}$ respectively. Also by Lemma 5.2,

$$
\Gamma\left(\mathbb{Z}_{p^{3}}\right)=\bar{K}_{p(p-1)} \nabla K_{p-1} .
$$




$$
\begin{gathered}
\operatorname{spec}^{S L}\left(\bar{K}_{p(p-1)}\right)=\left\{\begin{array}{cc}
0 & p(p-1) \\
1 & p^{2}-p-1
\end{array}\right\}, \\
\operatorname{spec}^{S L}\left(K_{p-1}\right)=\left\{\begin{array}{ll}
0 & 1-p \\
1 & p-2
\end{array}\right\} .
\end{gathered}
$$

Hence, the result follows from Theorem 3.1.

Theorem 5.6. For any prime $p$, the Seidel Laplacian spectrum of the zero-divisor graph $\Gamma\left(\mathbb{Z}_{p^{4}}\right)$ is

$$
\operatorname{spec}^{S L}\left(\Gamma\left(\mathbb{Z}_{p^{4}}\right)\right)=\left\{\begin{array}{cccc}
0 & 1-p^{3} & p^{3}-2 p^{2}+1 & p^{3}-2 p+1 \\
1 & p-1 & p^{2}-p-1 & p^{3}-p^{2}
\end{array}\right\} .
$$

Proof. The divisors of $p^{4}$ are $p, p^{2}, p^{3}$. The proper divisor graph of $p^{4}$ is the path $P_{3}$, in which $p \sim p^{3} \sim p^{2}$. The subgraph induced by $\mathbf{S}(p)$ is the null graph $\bar{K}_{p^{2}(p-1)}$, whereas the subgraphs induced by $\mathbf{S}\left(p^{2}\right)$ and $\mathbf{S}\left(p^{3}\right)$ are the complete graphs $K_{p(p-1)}$ and $K_{p-1}$ respectively. It can be seen that,

$$
\Gamma\left(\mathbb{Z}_{p^{4}}\right)=P_{3}\left[\bar{K}_{p^{2}(p-1)}, K_{p(p-1)}, K_{p-1}\right]
$$

Applying Corollary 5.1, we see that, $p^{3}-2 p+1, p^{3}-2 p^{2}+1,1-p^{3}$ are Seidel Laplacian eigenvalues of $\Gamma\left(\mathbb{Z}_{p^{4}}\right)$ with multiplicities $p^{3}-p^{2}-1, p^{2}-p-1$ and $p-2$ respectively. And the remaining three Seidel Laplacian eigenvalues of $\Gamma\left(\mathbb{Z}_{p^{4}}\right)$ are the eigenvalues of the matrix,

$$
T_{S L}\left(\Upsilon_{p^{4}}\right)=\left[\begin{array}{lcr}
p^{2}-2 p+1 & p-p^{2} & p-1 \\
p^{2}-p^{3} & p^{3}-p^{2}-p+1 & p-1 \\
p^{3}-p^{2} & p^{2}-p & p-p^{3}
\end{array}\right]
$$

It can be seen that, the above matrix has three eigen values, $\lambda_{1}=0, \lambda_{2}=1-p^{3}, \lambda_{3}=p^{3}-2 p+1$.

\section{Seidel signless Laplacian spectrum of the join of regular graphs}

We note that, each diagonal block in the Seidel signless Laplacian matrix of the join of two regular graphs is a symmetric matrix which bears an eigenvalue with all-one vector as the corresponding eigenvector, which facilitates the use of Fiedler's Lemma in the investigation of its spectrum. Since the main theorems of this section are in the same frame work of Fiedlers Lemma, we avoid repetition in proofs, except Theorem 6.1, where the concept of Schur complement and Coronal of a square matrix are incorporated.

Lemma 6.1. [19] Let $M, N, P, Q$ be matrices and let $\mathrm{M}$ be invertible. Let $S=\left[\begin{array}{cc}M & N \\ P & Q\end{array}\right]$, then $\operatorname{det} S=\operatorname{det} M . \operatorname{det}\left(Q-P M^{-1} N\right)$.

Lemma 6.2. [4] Let $A$ be an $n \times n$ matrix and $J_{n \times n}$ denote an all one matrix. Then,

$$
\operatorname{det}\left(x I_{n}-A-\alpha J_{n \times n}\right)=\left(1-\alpha \Gamma_{A}(x)\right) \cdot \operatorname{det}\left(x I_{n}-A\right),
$$

where $\alpha$ is a real number.

Theorem 6.1. Let $G_{i}$ be $r_{i}$-regular graph of order $n_{i}$, for $i=1,2$. The Seidel signless Laplacian polynomial of $G_{1} \nabla G_{2}$ is

$$
\Phi_{S L}^{+}\left(G_{1} \nabla G_{2} ; x\right)=\frac{\left(x-\kappa_{1}\right)\left(x-\kappa_{2}\right)-n_{1} n_{2}}{\left(x-\kappa_{1}\right)\left(x-\kappa_{2}\right)} \cdot \Phi_{S L}^{+}\left(G_{1} ; x+n_{2}\right) \cdot \Phi_{S L}^{+}\left(G_{2} ; x+n_{1}\right)
$$

where, $\kappa_{1}=2 n_{1}-n_{2}-4 r_{1}-2$ and $\kappa_{2}=2 n_{2}-n_{1}-4 r_{2}-2$.

Proof. Let $S L_{1}^{+}$and $S L_{2}^{+}$denote the Seidel signless Laplacian matrices of $G_{1}$ and $G_{2}$ respectively. Both $S L_{1}^{+}$and $S L_{2}^{+}$are symmetric and it can be seen that their row sums are the constants $2\left(n_{1}-2 r_{1}-1\right)$ and $2\left(n_{2}-2 r_{2}-1\right)$ respectively. Also

$$
\begin{aligned}
S L^{+}\left(G_{1} \nabla G_{2}\right) & =\left[\begin{array}{cc}
S L_{1}^{+}-n_{2} I_{n_{1}} & -J_{n_{1} \times n_{2}} \\
-J_{n_{1} \times n_{2}}^{T} & S L_{2}^{+}-n_{1} I_{n_{2}}
\end{array}\right] \\
& =\left[\begin{array}{cc}
S_{1} & -J_{n_{1} \times n_{2}} \\
-J_{n_{1} \times n_{2}}^{T} & S_{2}
\end{array}\right],
\end{aligned}
$$


where $S_{1}=S L_{1}^{+}-n_{2} I_{n_{1}}$ and $S_{2}=S L_{2}^{+}-n_{1} I_{n_{2}}$. Both $S_{1}$ and $S_{2}$ are symmetric and it can be easily seen that their row sums are the constants $\kappa_{1}=2\left(n_{1}-2 r_{1}-1\right)-n_{2}$ and $\kappa_{2}=2\left(n_{2}-2 r_{2}-1\right)-n_{1}$ respectively. Thus the coronal of $S_{1}$ and $S_{2}$ is found to be $\Gamma_{S_{1}}(x)=\frac{n_{1}}{x-\kappa_{1}}$ and $\Gamma_{S_{2}}(x)=\frac{n_{2}}{x-\kappa_{2}}$. Applying Lemma 6.2, and Lemma 6.1, it can be seen that

$$
\operatorname{det}\left(x I-S L^{+}\left(G_{1} \nabla G_{2}\right)\right)=\operatorname{det}\left(x I-S_{1}\right) \cdot \operatorname{det}\left(x I-S_{2}\right) \cdot\left(1-\Gamma_{S_{1}}(x) \Gamma_{S_{2}}(x)\right) \text {. }
$$

where $\Gamma_{S_{1}}(x)=\frac{n_{1}}{x-\left(n_{1}-2 r_{1}-1\right)}$ and $\Gamma_{S_{2}}(x)=\frac{n_{2}}{x-\left(n_{2}-2 r_{2}-1\right)}$.

Simplifying (10), we obtain the result.

As in section:4, Theorem 3.2 can be applied to find the Seidel signless Laplacian spectrum of the join of regular graphs. Consider $G\left[H_{1}, H_{2}, \ldots, H_{k}\right]$, where $G$ is a simple connected graph with vertices labeled as $1,2, \ldots, k$ with the Seidel matrix $S(G)=\left[s_{i j}\right]$ where $s_{i j}=-1$ if the vertices $i$ and $j$ are adjacent and $s_{i j}=1$ if the vertices $i$ and $j$ are not adjacent and $s_{i j}=0$ for the diagonal entries. Let $H_{j}$ be $r_{j}$ - regular and $\left|V\left(H_{j}\right)\right|=n_{j}$, for every $j=1,2, \ldots, k$. Let $S L^{+}\left(H_{j}\right)$ denote the Seidel signless Laplacian matrix of $H_{j},=1,2, \ldots, k$.

$$
S L^{+}\left(G\left[H_{1}, H_{2}, \ldots, H_{k}\right]\right)=\left[\begin{array}{cccc}
S L^{+}\left(H_{1}\right)+\tau_{1} I_{n_{1}} & s_{1,2} J_{n_{1} \times n_{2}} & \ldots & s_{1, k} J_{n_{1} \times n_{k}} \\
s_{1,2} J_{n_{1} \times n_{2}}^{T} & S L^{+}\left(H_{2}\right)+\tau_{2} I_{n_{2}} & \ldots & s_{2, k} J_{n_{2} \times n_{k}} \\
\vdots & & \ddots & \vdots \\
s_{1, k} J_{n_{1} \times n_{k}}^{T} & s_{2, k} J_{n_{2} \times n_{k}}^{T} & \ldots & S L^{+}\left(H_{k}\right)+\tau_{k} I_{n_{k}}
\end{array}\right]
$$

where $\tau_{j}=\sum_{i=1}^{k} s_{i j} n_{i}$.

Theorem 6.2. Consider $G\left[H_{1}, H_{2}, \ldots, H_{k}\right]$, where $G$ is a simple connected graph with vertices labeled as $1,2, \ldots, k$ and $S=\left[s_{i j}\right]_{k \times k}$ is the Seidel matrix of $G$ and $H_{j}$ is $r_{j}$-regular and $\left|V\left(H_{j}\right)\right|=n_{j}$, for every $j=1,2, \ldots, k$. Let $\left\{\sigma_{j 1}^{S L^{+}}=\right.$ $\left.2\left(n_{j}-2 r_{j}-1\right), \sigma_{j 2}^{S L^{+}}, \ldots, \sigma_{j n_{j}}^{S L^{+}}\right\}$be the Seidel signless Laplacian eigenvalues of $H_{j}$, for $j=1,2, \ldots, k$. Then, the Seidel signless Laplacian spectrum of the $G$-join of the graphs $H_{1}, H_{2}, \ldots, H_{k}$ is given by,

$$
\operatorname{spec}^{S L^{+}}\left(G\left[H_{1}, H_{2}, \ldots, H_{n}\right]\right)=\left(\bigcup_{j=1}^{k} \bigcup_{i=2}^{n_{j}}\left(\sigma_{j i}^{S L^{+}}+\tau_{j}\right)\right) \cup \operatorname{spec}\left(T_{S L^{+}}(G)\right)
$$

where $\tau_{j}=\sum_{i=1}^{k} s_{i j} n_{i}$ and

$$
T_{S L^{+}}(G)=\left[\begin{array}{cccc}
2\left(n_{1}-2 r_{1}-1\right)+\tau_{1} & s_{1,2} n_{2} & \ldots & s_{1, k} n_{k} \\
s_{1,2} n_{1} & 2\left(n_{2}-2 r_{2}-1\right)+\tau_{2} & \ldots & s_{2, k} n_{k} \\
\vdots & \vdots & \ddots & \vdots \\
s_{1, k} n_{1} & s_{2, k} n_{2} & \ldots & 2\left(n_{k}-2 r_{k}-1\right)+\tau_{k}
\end{array}\right]
$$

Proof. Since $H_{j}$ is $r_{j}$-regular, $S L^{+}\left(H_{j}\right)=\left(n_{j}-2 r_{j}-1\right) I_{n_{j}}+\left(S\left(H_{j}\right)\right)$, where $S\left(H_{j}\right)$ is the Seidel matrix of $H_{j}$. By lemma 3.2, $n_{j}-2 r_{j}-1$ is a Seidel eigenvalue of $H_{j}$ with corresponding eigenvector $\mathbf{1}_{n_{j}}$. Thus $S L^{+}\left(H_{j}\right)$ has eigenvalue $2\left(n_{j}-2 r_{j}-1\right)$ with corresponding eigenvector $\mathbf{1}_{n_{j}}$, for every $j=1,2, \ldots, k$. Hence, as evident from (11), the $j^{t h}$ diagonal block of the Seidel signless Laplacian matrix of $G\left[H_{1}, H_{2}, \ldots, H_{n}\right]$ is the symmetric matrix $S L^{+}\left(H_{j}\right)+\tau_{j} I_{n_{j}}$ which has an eigenvalue $2\left(n_{j}-2 r_{j}-1\right)+\tau_{j}$ with eigenvector $\mathbf{1}_{n_{j}}$, for $j=1,2, \ldots, k$. Thus, taking

$$
M_{j}=S L^{+}\left(H_{j}\right)+\tau_{j} I_{n_{j}}, \quad\left(\alpha_{i_{j}, j}, \mathbf{u}_{i_{j}, j}\right)=\left(2\left(n_{j}-2 r_{j}-1\right)+\tau_{j} \quad, \quad \frac{1}{\sqrt{n_{j}}} \mathbf{1}_{n_{j}}\right)
$$

and the real numbers

$$
\rho_{l, q}=s_{l q} \sqrt{n_{l} n_{q}}
$$

for $l \in\{1,2, \ldots, k-1\}, q \in\{l+1, \ldots, k\}$, the result follows from Theorem 3.2.

Example 6.1. Consider the zero-divisor graph $\Gamma\left(\mathbb{Z}_{p^{2} q}\right)$, where $p$ and $q$ are distinct primes. The proper divisors of $p^{2} q$ are $d_{1}=p, \quad d_{2}=q, \quad d_{3}=p^{2}, \quad d_{4}=p q$. The proper divisor graph of $p^{2} q$ is the path $P_{4}$, where $p \sim p q \sim p^{2} \sim q$. The subgraphs induced by $\mathbf{S}(p), \mathbf{S}(q), \mathbf{S}\left(p^{2}\right), \mathbf{S}(p q)$ are $\bar{K}_{(p-1)(q-1)}, \bar{K}_{p(p-1)}, \bar{K}_{(q-1)}$ and $K_{p-1}$ respectively. Hence,

$$
\Gamma\left(\mathbb{Z}_{p^{2} q}\right)=P_{4}\left[\bar{K}_{(p-1)(q-1)}, \bar{K}_{p(p-1)}, \bar{K}_{(q-1)}, K_{p-1}\right]
$$


Applying Theorem 6.2, we see that, the Seidel Laplacian spectrum of $\Gamma\left(\mathbb{Z}_{p^{2} q}\right)$ is the multi set $\left\{\begin{array}{cccc}p^{2}+p q-3 p-1 & p^{2}+p q-p-2 q-1 & p q-p^{2}-p-1 & p^{2}-p q-p+3 \\ p q-p-q & p^{2}-p-1 & q-2 & 3-p\end{array}\right\}$ together with the spectrum of the matrix,

$$
\begin{gathered}
T_{S L^{+}}\left(\Upsilon_{p^{2} q}\right)= \\
{\left[\begin{array}{cccc}
p^{2}+2 p q-4 p-q & p^{2}-p & q-1 & 1-p \\
(p-1)(q-1) & 2 p^{2}+p q-2 p-2 q-1 & 1-q & p-1 \\
(p-1)(q-1) & p-p^{2} & p q-p^{2}-p+q-2 & 1-p \\
-(p-1)(q-1) & p^{2}-p & 1-q & p^{2}-p q-2 p+4
\end{array}\right] .}
\end{gathered}
$$

\section{Conclusion}

The induced subgraphs which are either cliques or null graphs, shape the combinatorial structure of the zero divisor graph on the ring $\mathbb{Z}_{n}$. The all-one matrix blocks constituting the matrix described in this paper, facilitates the use of Fiedler's lemma in the computation of the spectrum.

\section{REFERENCES}

[1] Van Lint J.H., Seidel J.J., “Equilateral point sets in elliptic geometry”, Indag.Math., Vol.28, pp. 335-348, 1966.

[2] H.S. Ramane, R.B. Jummannaver, I. Gutman, "Seidel Laplacian energy of graphs", Int.J.Appl.Graph Theory, Vol.1, No.2, pp. 74-82, 2017.

[3] Robert. L. Hemminger, “The Group of an $X$-join of Graphs”, Journal of Combinatorial Theory, Vol.5, pp. 408-418, 1968.

[4] X. Liu, Z.Zhang, "Spectra of subdivision-vertex join and subdivision-edge join of two graphs", Bull. Malays.Math.Sci.Soc., Vol.42, pp. 15-31, 2019.

[5] I. Beck, "Coloring of a commutative ring”, J. Algebra, Vol.116, No.1, pp. 208-226, 1988.

[6] D.F. Anderson and P.S. Livingston, "The zero-divisor graph of a commutative ring”, J. Algebra, Vol.217, No.2, pp. 434-447, 1999.

[7] Mulay S.B., "Cycles and symmetries of zero-divisors”, Comm. Algebra, Vol.30, No.7, pp. 3533-3558, 2007.

[8] P.M. Magi, Sr. Magie Jose, Anjaly Kishore, "Adjacency matrix and eigenvalues of the zero divisor graph $\Gamma\left(\mathbb{Z}_{n}\right)$ ", J. Math. Comput. Sci., Vol.10, pp. 1285-1297, 2020.

[9] P.M. Magi, Sr. Magie Jose, Anjaly Kishore, "Spectrum of the zero-divisor graph on the ring of integers modulo n", J. Math. Comput. Sci., Vol.10, pp. 1643-1666, 2020.

[10] S. Chattopadhyay, K.L. Patra, B.K. Sahoo, "Laplacian eigenvalues of the zero divisor graph of the ring Zn", Linear Algebra and its applications, Vol.584, pp. 267-286, 2020.

[11] H.Kumar, K.L. Patra, B.K.sahoo, "Proper divisor graph of a positive integer”, arXiv.2005.O4441v1[math.Co]May 2020.

[12] H.S. Ramane, K.Ashoka, D.Path, "On the Seidel Laplacian and Seidel Signless Laplacian Polynomial of Graphs”, Kyungpook Math.J., Vol.61, pp. 155-168, 2021.

[13] M.Fiedler, "Eigenvalues of nonnegative symmetric matrices", Linear Algebra appl., Vol.9, pp. 119-142, 1974.

[14] Andries E. Brouwer, Willem H. Haemers, "Graphs with few eigenvalues” in Spectra of Graphs, Springer, New York, 2012, pp.215-216.

[15] D.M.Cardoso, Maria Aguieiras A. de Freita, E.A. Martin, Maria Robbiano, "Spectra of graphs obtained by a generalization of the join graph operation", Discrete Mathematics, Vol.313, pp. 733-741, 2013.

[16] P. M. Magi, Sr. Magie Jose, A.Kishore, "Distance and distance Laplacian spectrum of the Zero-divisor Graph on the ring of integers modulo n", Advances in Mathematics: Scientific Journal, Vol. 9, no.12, pp. 10591-10612, 2020.

[17] F.R.K. Chung, Robert P. Langlands, "A combinatorial Laplacian with vertex weights", Journal of Combinatorial Theory, series A No.75, pp. 316-327, 1996.

[18] R.A. Horn, C.R. Johnson, "Location and perturbation of Eigenvalues" in Matrix Analysis, second edition, Cambridge University Press, 2013, pp. 392-393.

[19] F. Zhang, "Historical Introduction" in The Schur complement and its application, Springer,2005, pp. 14-17. 